\title{
Einiges über den Röntgenbetrieb bei den vorderen Sanitätsformationen.
}

Von Dr. Otto Loewe, Chirurg an einem Feldlazarett.

In der feldärztlichen Literatur nehmen die röntgenologischen Arbeiten einen breiten Raum ein, einen so breiten, daß die Fülle des wissenschaftlich Beachtenswerten den nicht spezialistisch vorgebildeten Feldarzt verwirren und ihm die Einrichtung eines für die vorderen Formationen geeigneten einfachen und doch leistungsfähigen Röntgenlaboratoriums erschweren muß. Konnte doch bereits im Frühling dieses Jahres Grashey auf der Kriegschirurgentagung über 150 Methoden der Fremdkörperlokalisierung berichten, und inzwischen ist das zweite Hundert sicher reichlich voll geworden.

Es soll deshalb nicht Aufgabe dieser Arbeit sein, über neue Methoden zu berichten, sondern sie soll darlegen, wie es gelingt, unter eklektischer Verwertung der literarischen Anregungen behelfsmäBige Vorrichtungen zu schaffen, die es erlauben, allen im Feldlazarett vorkommenden Indikationen auf dem Gebiet der Röntgenologie gerecht zu werden.

Die erste Bedingung für gute Erfolge bei röntgenologischen Arbeiten ist eine tadellose Verdunklungsvorrichtung für den Unter. suchungsraum. So selbstverständlich diese Forderung ist, so oft wird sie auch in sonst sachgemäß eingerichteten Lazaretten vernachlässigt. Gewiß ergeben sich ja auch oft Interessenkonflikte, wenn man daran gehen will, den mit möglichster Lichtfülle bedachten Operationsraum, der in Anbetracht der häufigen Fremdkörper-Operationen unter Röntgenlicht am besten auch als Röntgenzimmer dient, mit exakt arbeitenden Verdunklungsvorrichtungen zu versehen. Aber erster Grundsatz muß bleiben: ohne absolute Dunkelheit kein röntgenologisches Arbeiten. Denn bei der Durchleuchtung gewinnt man ein lebendes, plastisches Röntgenbild, die Platte gibt nur tote Schatten. (Fig, 1.)

Auf der vorangestellten Abbildung ist unser wichtigstes röntge nologisches Hilfsge rät veranschaulicht. Es besteht lediglich aus einer Aufhängevorrichtung für eine Tragbahre, ersetzt uns aber vollkommen das Trochoskop, vor dem es für den Feldbetrieb sogar den Vorzug hat, eine Untersuchung des Verletzten ohne Lagerungswechsel auf "seiner Transportbahre zu gestatten. Jeder Verwundete, der einen Steck. schu $B$ in einer der drei Körperhöhlen oder einen Knochenbruch hat, wird sofort bei der Ankunft auf seiner Transportbahre über der Röntgenröhre aufgehängt, der ebenso wie die auf der Abbildung nicht gezeichnete Blende am gleichen Stativ wie die Röhre befestigte Leuchtschirm in die richtige Lage gebracht und der Körper des Verletzten abgeleuchtet. Bei dieser Ableuchtung wird jedoch nicht, wie beim Trochoskop, Klinoskop und ähnlichen Röntgentischen, Röhre und Leuchtschirm verschoben, sondern in einfachster Weise auf vorher festgelegtes Kommando hin durch die beiden an den Handgriffen der Bahre postierten Wärter die 
schwebende Bahre nach oben, unten, rechts, links oder den Zwischenlagen in Bewegung gesetzt und dann wieder auf Kommando in der gewünschten Stellung fixiert. Auf diese Weise lassen sich die großen Körperhöhlen oder auch große

Teile der Extremitäten ohn
Verschiebung von Röhre, Schirm oder Patientableuchten. Arzt und Wärter sind dabei durch vorgesetzte kleine, eiserne Ofenschirme, die auf der Abbil. dung der Uebersichtlichkeit wegen, ebenso wie die das störende Licht abfangende schwarze Röhrenklappe, nicht gezeichnet

sind, einigerma Ben geschützt. Auch tritt die in der Feldröntgenausrüstung meist enthaltene Bleigummisch ürze in Funktion. Trotzdem tut man gut diesen Dienst von sämtlichen Sanitätsmannschaften der Formation abwechselnd versehen zu lassen, um mit Sicherheit jede Schädigung des Personals durch Röntgenstrahlen zu vermeiden. Muß man häufiger mit den gleichen Leuten arbeiten, so schützt man besser die Haut dadurch, $\mathrm{da} ß$ vier große Konservenbüchsen oder auch eiserne Kochtöpfe mit gelochtem Boden über die Holben der Trage geschoben werden, in die dann die Helfer mit ihren Händen hineinschlüpfen.. Der Arzt fordere zum Schutz seiner Hände strahlendichte Handschuhe an, für die dann zum Gebrauch bei Operationen unter Röntgenlicht noch sterilisierbare Ueberhandschuhe aus Leinenstoff anzufertigen wären.

Die gleiche Einrichtung dient auch als Tisch für die Operationen im Röntgenlicht, die wir nach einer später noch zu exläuternden, sehr einfachen Methode ausführen. Doch empfiehlt es sich, für diese Fälle eine besondere, aus Holz und. Segeltuch angefertigte Tragbahre, die für Operationszwecke vorbehalten ist, zu benutzen, einerseits der Sauberkeit wegen, dann aber auch, um das lästige Ueberspringen von Funken zu den Metallteilen der planmäßigen Tragen zu vermeiden.

Daß dieser Tragbahrentisch bei Verwendung von Plattenkassetten auch als Aufnahmetisch dienen kann, ist selbstverständlich. Er läßt sich aber auch bei Anwendung der Untertisçhröhre für Aufnahmen von unten nach oben verwenden, wenn man nämlich die Kassette an dem bei Durchleuchtungen den Schirm tragenden, am .Röntgenstativ verschieblichen Arm befestigt. Auch hierbei ist die Benutzung der am gleichen Stativ befindlichen, auswechselbaren Blende nicht zu vernachlässigen.

Für die stationären Lazarette des Stellungskriegs, für Kriegslazarette, überhaupt für alle Sanitätsanstalten des Feldheeres, in denen der Röntgenapparat auoh intern medizinischen $Z$ wecken, vor allem der für die Entscheidung über Kriegsverwendungsfähigkeit so überaus wichtigen Feststellung der Herzgröße dienen soll, sei dio Anfertigung eines etwas komplizierteren Tisches empfohlen. Er ist jedoch in seiner Konstruktion so einfach gehalten, daß ihn die wohl bei jeder Formation zu findenden manuell geschickten Leute anfertigen können, auch wenn eigentliche Präzisionshandwerker fehlen, und das auch dann, wenn man den Tisch mit der später beschriebenen orthodiagraphischen Einrichtung versehen läßt.

Dieser Lager u ngsa p parat (Fig. 2) besteht aus einem gewöhnlichen Tischgestell in den üblichen Operationstischmaßen, das etwa $20 \mathrm{~cm}$ über der Erde einen quer verlaufenden Boden aus glattem, hartem Material, wenn irgend möglich, aus Glas oder Marmor trägt. Doch braucht nicht der ganze Boden aus diesem wertvollen Stoffen zu bestehen, es 'genügt, wenn eine solche Platte in der Größe $80: 50 \mathrm{~cm}$ bündig in die Mitte einer Holzplatte eingearbeitet wird. Als Tischplatte dient wiederum eine gewöhnliche Tragbahre aus Holz und Segeltuch, damit auch bei der Benutzung dieses Tisches der Patient ohne Umlagerung vom Bett zum Röntgen und wieder in das Bett befördert werden kann. Auf der Marmorplatte steht auf drei gut gearbeiteten und präzis funktionierenden Kugelrollen; die als Eckpunkte eines möglichst kleinen Dreiecks angeordnet sind, ${ }^{1}$ ) ein massiver Holzkasten, der eine Lagerungsvorrichtung für die mit der Antikathode nach oben schauende Röntgenröhre enthält. In den Deckel ist eine auswechselbare Metallblende eingepaßt. An der einen Längswand des Kastens ist eine vertikal nach oben gerichtete, etwa $1 / \frac{1}{2} \mathrm{~m}$ lange

1) Die Rollen sind auf der Abbildung irrtümlich an den Kastenocken gezeichnet.
Stange befestigt, an der, durch eine Schraube fixierbar, ein zur Aufnahme des Leuchtschirms oder der Plattenkassette dienender Rahmen gleitet. Kasten und Schirmträger sind gemeinsam durch einen am letzteren angebrachten, strahlen-

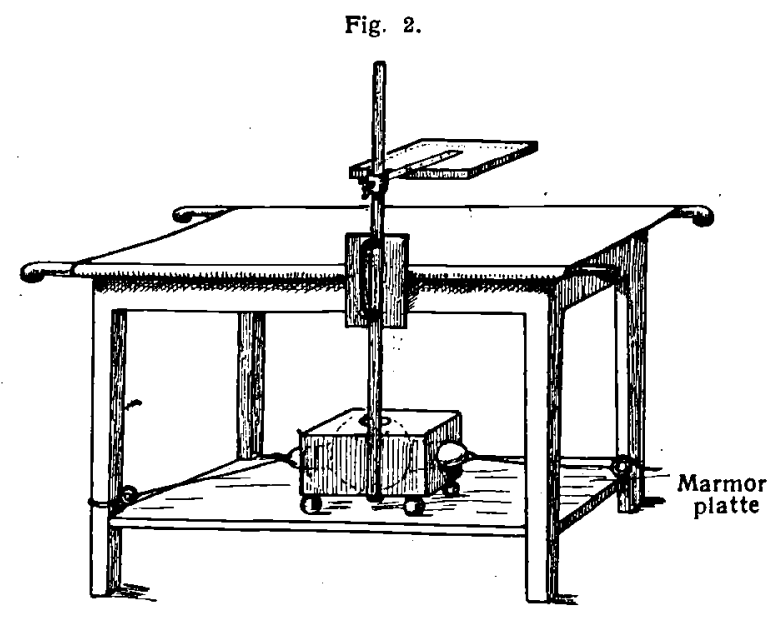
gesch ützten Handgriff mit Hilfe der Kugelrollen auf der glat. ten Tragfläche spielend leicht $\mathrm{zu}$ verschieben, so leicht, $\mathrm{da} B$ man mit einem am Boden des Leuchtschirms angebrachten Stift einen kleinen Kreis beschreiben

bzw. eine Herzgrenze umreißen kann. Um ein Kippen des Gestells zu verhüten, muß der Boden und die der Tragseite gegenüberliegende Längsseite des Röhrenkastens durch eine Steinplatte belastet sein.

Der schon erwähnte, in Abb. 3 in einer schematischen Zeichnung seines Durchschnitts wiedergegebene Orthodiagraph ist von der denkbarsten Einfachheit. Er besteht nur aus einem in einer Führung gleitenden, aus mög:

lichst schwerem

Metall, am bes. ten aus Blei gefertigten Stift, der an seinem Ende einen winzigen Watte bausch zur Auf nahme von Tu. sche trägt. Die Führungshülse

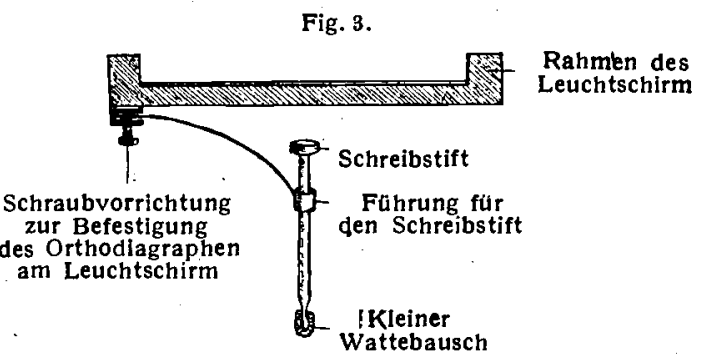

ist mit dem Rah-

men des Leuchtschirms durch einen gebogenen Metallstab in Verbindung gebracht. Die Benutzung des Orthodiagraphen geschieht in der Weise, daß zunächst mit Hilfe eines Senkbleis der Stift im. Zentralstrahl des Röntgenlichtes eingestellt wird. Ueber dem Brustkorb 'des Patienten wird ein mit Papier bespannter Rahmen befestigt, auf dem dann der mit Tusche befeuchtete Wattebausch dadurch die Umrisse des Herzens aufzeichnet, daß der Schatten des den Stift krönenden Knopfes dem Rande des Herzschattens entlang geführt wird. Auch auf dem Körper des Patienten selbst kann man zeichnen, denn der in der Führung gleitende Stift paßt sich allen Niveauunterschieden ohne weiteres an.

Gewiß gehört ein Orthodiagraph nicht zu den notwendigen Hilfs. mitteln einer Sanitätsformation der vorderen Linie. Aber das von mir angegebene Modell ist so einfach herzustellen, daß vielleicht mancher klinisch interessierte Arzt es in den ruhigen Zeiten gern benutzt, um Studien über akute Dilatationen bei Soldaten der Front zu machen und Herzveränderungen festzustellen, die bei der Ankunft der Leute in den rückwärtigen Lazaretten sich längst wieder ausgeglichen haben.

Die beiden abgebildeten Lagerungsapparate dienen, wie erwähnt, auch als Operationstische für Eingriffe unter Röntgenlicht, das wir bei jedem Fremdkörper zu Hilfe nehmen, den man nicht ohne weiteres unter der Haut fühlen kann. Wir gehen dabei nach folgender Methode vor: EB werden zunächst am Leuch tschirm zwei gerade Linien bestimmt, in deren Schnittpunkt der Fremdkörper liegt, und zwar so, daß wir das Schattenbild beider Tastkugeln eines gewöhnlichen Tasterzirkels auf dem Leuchtschirm mit dem Schatten des Fremdkörpers zur Deckung bringen, soda $B$ also nur ein Schattengebilde zu sehen ist. Die Lagepunkte der Tastkugeln werden alsdann mit dem Dermatographen auf der Haut bezeichnet. An einer anderen Stelle der Zirkumferenz des getroffenen Organs, aber etwa in gleicher Höhe, wird der gleiche Vorgang wiederholt und wiederum eine Markierung angebracht. Denken wir uns nun die markíerten Punktpaare durch Verbindungslinien miteinander vereinigt, so muß der Fremdkörper genau im Schnittpunkt beider Linien liegen. Nachdem wir une so ungefähr über die Lage des Fremdkörpers informiert haben, wird bei Tageslicht ein kleiner Schnitt bis etwa in die Tiefe gemacht, wo wir den Fremdkörper lokalisiert haben. Sehen oder fühlen wir ihn dann nicht sofort, so verzichten wir auf weiteres präparatorisches Suchen und führen eine $\mathbf{K}_{0}$ che rsche oder Koeberle sche Klemme in den tiefsten Punkt der 
Wunden ein. Unter Röntgenlicht suchen wir dann den Schatten des Fremd. körpers "zwischen die Schatten der Schieberbranchen zu bringen und unter Führung"des Auges den Fremdkörper zu fassen, was meist überraschend leicht gelingt. Mit einigen vorsichtigen hebelnden Bewegungen wird dann der Fremdkörper fast immer ohne Schwierigkeit herausbefördert. Gelingt das bei einem schon seit langem eingekapselten Fremdkörper nicht, so hält man ihn mit der Klemme fest, macht hell und legt mit einigen Schnitten den fixierten Fremdkörper bloß.

Das Verfahren empfiehlt sich besonders bei kleinen Fremdkörpern, an denen man sich ja präparierend leicht vorbeiarbeitet, und hat sich mir schon im Frieden bei der Entfernung von Nadelteilen wohl bewährt. $\mathrm{Zu}$ widerraten ist es freilich in der nächsten Nähe der großen Gefäße und Nerven, die ja versehentlich gezerrt oder angerissen werden könnten, doch läßt sich bei der vorherigen Orientierung auf dem Leuchtschirm fast immer ein Weg finden, auf dem man diese empfindlichen Teile umgehen kann. Auch bei Hirnsteckschüssen kann die Methode versucht werden, wenn man den Fremdkörper im Röntgenlicht ganz deutlich sieht, aber auch dann nur nach gründlichem Durchdenken der anatomischen Lage des Falles, wenn man sicher ist, daß die Zange auf ihrem Weg nicht etwa die Ventrikel, vor allem nicht die Gegend des vierten berührt. Aber unter diesen Voraussetzungen und einem der Empfindlichkeit des Gewebes entsprechenden zarten Handhaben der Zange ist das Vorgehen sicher weniger eingreifend als ein Suchen mit dem Finger und kaum verletzender, aber im Erfolg sicherer als eine Extraktion mit dem Riesenmagneten, zumal dieser ja überhaupt nur bei eisenhaltigen Splittern anwendbar und bei einer Geschoßlage in der Nähe der lebenswichtigen Zentren ebenfalls höchst bédenklich ist.

Anwendbar ist die Methode natürlich nur in Fällen, wo man den Fremdkörper im Röntgenlicht zu Gesicht bekommen kann; aber das wird um so häufiger der Fall sein, je besser Verdunklungsvorrichtung und Blendentechnik funktionieren. Sieht man den Fremdkörper nicht, dann ist natürlich eine stereoskopische Aufnahme oder eine der zahlreichen Tiefenberechnungs- oder M̀essungsmethoden heranzuziehen. Lediglich auf Grund von zwei Aufnahmen in den beiden Ebenen an die Entfernung eines Fremdkörpers herangegangen zu sein, hat wohl jeder von uns schon des öfteren bitter bereut.

Zum Schluß noch einige Worte über die Asepsis bei den 0 perationen unter Röntgenlicht. Grundsatz dabei ist: Das Röntgenen darf die Asepsis, die Asepsis den Röntgenbetrieb nicht stören. Daher ist unbedingt getrenntes Personal für die beiden $Z$ weige der operativen Röntgenologie nötig, jeder Mitwirkende muB seine Funktionen kennen und diese, aber nur diese, nach kurzem Kommandowort ausüben. Für die exakte Asepsis sind, abgesehen von den üblichen sterilen Tüchern, die erwähnten leinenen Fausthandschuhe für Operateur und Assistenten und Tücher mit Knopfvorrichtungen zur Umhüllung der Unterfläche des Leuchtschirms erforderlich, der in ständigerer Berührung mit Händen und Instrumenten ist. Der Gang der Handlung ist der, daß der Patient operationsfertig gemacht, aber noch nicht steril bedeckt wird, dann wird die Lokalisation des Fremdkörpers vorgenommen, und erst nach dieser sterilisieren "sich Operateur und Gehilfen, machen den beschriebenen Einschnitt, führen 'die Klemme ein, grenzen das Operationsgebiet mit Tüchern zu einer sterilen Kammer ab, in die nur die Hände des'Operateurs hund, wenn es Haken halten gibt, auch die des Assistenten hineinschlüpfen. ! Erst dann wird das Röntgenlicht 'eingeschaltet. So sind alle Voraissetzungen zum Erfolg gegeben, nämlich gute 'Asepsis, exakte Lokalisation und ein sicheres Extraktionsverfahren. 